\title{
New Oral Anticoagulants versus Warfarin for Cerebral Venous Thrombosis: A Multi-Center, Observational Study
}

\author{
Mohammad Wasay, ${ }^{\mathrm{a}}$ Maria Khan, ${ }^{\mathrm{b}}$ Haris Majid Rajput, ${ }^{\mathrm{c}}$ Salman Farooq, ${ }^{\mathrm{a}}$ Mohammed Ibrahim Memon, ${ }^{\mathrm{d}}$ \\ Suhail Abdulla AlRukn, ${ }^{\mathrm{b}}$ Abdul Malik, ${ }^{\mathrm{e}}$ Foad Abd-Allah, Raja Farhat Shoaib, ${ }^{\mathrm{g}}$ Rizwana Shahid, \\ Sadia Nishat, ${ }^{i}$ Safia Awan ${ }^{j}$ \\ aDepartment of Neurology, Aga Khan University, Karachi, Pakistan \\ bDepartment of Neurology, Rashid Hospital, Dubai, United Arab Emirates \\ 'Department of Neurology, Pakistan Institute of Medical Sciences, Islamabad, Pakistan \\ 'Department of Neurology, King Fahad Medical City, Riyadh, Kingdom of Saudi Arabia \\ 'Department of Medicine, Liaquat College of Medicine and Dentistry, Karachi, Pakistan \\ fDepartment of Neurology, Cairo University, Cairo, Egypt \\ ${ }^{9}$ Department of Neurology, Shifa International University and Hospital, Islamabad, Pakistan \\ hDepartment of Neurology, Imam Abdulrahman Bin Faisal University, Dammam, Kingdom of Saudi Arabia \\ 'Department of Neurology, Sindh Institute of Urology and Transplantation, Karachi, Pakistan \\ 'Department of Medicine, Aga Khan University, Karachi, Pakistan
}

\section{Dear Sir:}

The incidence of cerebral venous thrombosis (CVT) is on the rise, partly due to an improvement in the diagnostic techniques.' However, the therapeutic options have not changed much in the past three decades. Heparin followed by vitamin $\mathrm{K}$ antagonist (VKA), Warfarin is still the mainstay of treatment, ${ }^{2-4}$ although the limitations of VKA therapy including risk of major bleeding, need for constant monitoring, and drug and dietary interactions are all well recognized. ${ }^{5,6}$

New direct oral anticoagulants (NOACs), which are target specific for factor Xa (rivaroxaban, apixaban, edoxaban) or thrombin (dabigatran), were developed to overcome some of these limitations. These drugs have already shown to be safer then VKA for recurrent venous thromboembolism and embolism prevention in atrial fibrillation, with fewer intracranial and extracranial hemorrhages, fewer fatal hemorrhages and no increase in the risk of gastrointestinal hemorrhages. 'Based on this safety data, and the efficacy results from venous thromboembolism studies, there is a growing tendency for neurologists to consider them for treatment of CVT. A few case series have been published supporting the safety and efficacy of these drugs in CVT cases. ${ }^{8-11}$ More re- cently, the results of one randomized controlled trial (randomized controlled trial of the safety and efficacy of dabigatran etexilate vs. dose-adjusted warfarin in patients with cerebral venous thrombosis [RESPECT CVT]) were presented at World Stroke Congress in 2018. ${ }^{12}$ The trial randomized 120 patients to either dabigatran or warfarin and showed no recurrence of venous thrombotic events and a small number of major bleedings in both arms.

Before the results of RESPECT CVT were made available, we conducted a multicenter prospective, observational study to evaluate the safety of NOACs compared to warfarin in patients with CVT. Data was collected from October 2016 to October 2018. Nine centers in four countries Pakistan, Saudi Arabia, Egypt, and the United Arab Emirates participated in the study. All nine centers are tertiary care hospitals with full time neurologists and availability of diagnostic modalities for the diagnosis of CVT.

We included consecutive patients aged 18 years or more, who presented with clinical features of $\mathrm{CVT}$, confirmed with venography (either computed tomography [CT] or magnetic resonance [MR]). Those with traumatic or septic CVT were excluded. Baseline characteristics including demographics, Glasgow Coma Scale, clinical features, imaging findings, and anticoagulant at discharge were recorded. Choice of oral anticoagulation was left 
Table 1. NOACs vs. warfarin for cerebral venous thrombosis $(n=111)$

\begin{tabular}{|c|c|c|c|c|}
\hline Variable & All patients $(n=111)$ & NOACs $(n=45,41 \%)$ & Warfarin $(n=66,59 \%)$ & $P$ \\
\hline Age $(y r)$ & $39.3 \pm 14.9$ & $36.5 \pm 14.7$ & $41.3 \pm 14.8$ & 0.10 \\
\hline Female sex & $64(57.7)$ & $27(60)$ & $37(56.1)$ & 0.68 \\
\hline \multicolumn{5}{|l|}{ Predisposing factors } \\
\hline Pregnancy/puerperium & $15(23.1)$ & $8(28.6)$ & $7(18.9)$ & 0.36 \\
\hline Hematological disorders & $74(66.7)$ & $28(62.2)$ & $46(69.7)$ & 0.41 \\
\hline Malignancy & $6(5.4)$ & $0(0)$ & $6(10.5)$ & 0.03 \\
\hline Systemic infections & $36(32.4)$ & 15 (33.3) & $21(31.8)$ & 0.86 \\
\hline Drugs/OCP & $11(9.9)$ & $4(9)$ & $7(11)$ & 0.32 \\
\hline Other medical conditions & $7(6.3)$ & $6(13.3)$ & $1(1.5)$ & 0.01 \\
\hline \multicolumn{5}{|l|}{ Clinical findings } \\
\hline Baseline GCS (available in 89 cases) & & & & 0.73 \\
\hline $13-15$ & $64(71)$ & $28(74)$ & $36(70)$ & \\
\hline $9-12$ & $17(19)$ & $7(18)$ & $10(20)$ & \\
\hline$\leq 8$ & $8(10)$ & $3(8)$ & $5(10)$ & \\
\hline Seizures & $37(33.3)$ & $14(31)$ & $23(34.8)$ & 0.68 \\
\hline Paresis & $49(44)$ & $22(48)$ & $27(52)$ & 0.32 \\
\hline Aphasia & $7(6.3)$ & $5(11)$ & $2(3.0)$ & 0.08 \\
\hline Headache & $84(75.7)$ & $42(93)$ & $42(63.6)$ & $<0.001$ \\
\hline Papilledema & $4(3.6)$ & $1(2)$ & $3(4)$ & 0.18 \\
\hline \multicolumn{5}{|l|}{ Imaging findings } \\
\hline Intracerebral hemorrhage & $45(40.5)$ & $25(55.6)$ & $20(30.3)$ & 0.01 \\
\hline Venous infarction & $54(48.6)$ & $25(55.6)$ & $29(43.9)$ & 0.22 \\
\hline Midline shift & $10(9.0)$ & $4(8.9)$ & $6(9.1)$ & 0.97 \\
\hline Hydrocephalus & $1(0.9)$ & $0(0)$ & $1(1.5)$ & 0.40 \\
\hline SSS thrombus & $56(50.5)$ & $26(57.8)$ & $30(45.5)$ & 0.20 \\
\hline TS thrombus & $60(54.1)$ & $30(66.7)$ & $30(45.5)$ & 0.02 \\
\hline Straight sinus/DCVT/cortical vein & $28(25.2)$ & $11(24.4)$ & $17(25.8)$ & 0.87 \\
\hline Sigmoid sinus/jugular vein & $62(55.9)$ & $29(64.4)$ & $33(50)$ & 0.13 \\
\hline \multicolumn{5}{|l|}{ Treatment } \\
\hline Heparin/LMWH & $79(71)$ & $36(80)$ & $43(65)$ & 0.06 \\
\hline Surgery & $12(10.8)$ & $5(12.5)$ & $7(11.7)$ & 0.90 \\
\hline Start of oral anticoagulation in days & $6(3-11)$ & $7(3-12)$ & $5(3-10)$ & \\
\hline $\mathrm{mRS}$ at discharge & 111 & 45 & 66 & 0.52 \\
\hline 0 & $23(21)$ & $11(25)$ & $12(18)$ & \\
\hline 1 & $29(26)$ & $11(23)$ & $18(28)$ & \\
\hline 2 & $28(25)$ & $12(27)$ & $16(23)$ & \\
\hline 3 & $15(14)$ & $4(9)$ & $11(17)$ & \\
\hline 4 & $10(10)$ & $5(10)$ & $5(8)$ & \\
\hline 5 & $2(2)$ & $1(2)$ & $1(2)$ & \\
\hline 6 & $4(4)$ & $1(2)$ & $3(5)$ & \\
\hline
\end{tabular}


Table 1. Continued

\begin{tabular}{|c|c|c|c|c|}
\hline Variable & All patients $(n=111)$ & NOACs $(n=45,41 \%)$ & Warfarin $(n=66,59 \%)$ & $P$ \\
\hline $\mathrm{mRS}$ at 6 months & 95 & 39 & 56 & 0.30 \\
\hline 0 & 37 (39) & $17(44)$ & $20(36)$ & \\
\hline 1 & $23(24)$ & $8(21)$ & $15(28)$ & \\
\hline 2 & $19(20)$ & $10(26)$ & $9(16)$ & \\
\hline 3 & $9(10)$ & $2(4)$ & $7(12)$ & \\
\hline 4 & $4(4)$ & $1(2)$ & $3(5)$ & \\
\hline 5 & $3(3)$ & $1(2)$ & $2(3)$ & \\
\hline 6 & $2(0.02)$ & $1(0.03)$ & $1(0.02)$ & \\
\hline
\end{tabular}

Values are presented as mean \pm standard deviation, number (\%), or median (interquartile range).

NOAC, new direct oral anticoagulant; OCP, oral contraceptive pill; GCS, Glasgow Coma Scale; SSS, superior sagittal sinus; TS, transverse sinus; DCVT, deep cerebral vein thrombosis; LMWH, low molecular weight heparin; mRS, modified Rankin Scale.

to the discretion of the treating physician. Recruited patients were followed up prospectively for outcomes assessment which was done by the treating physician in outpatient clinic setup. Treating physicians took verbal consent from the patients or their surrogates for contributing their data to the study. Ethics approval was taken from the Institutional Review Board of each participating center. Data was entered and analyzed on SPSS version 21 (IBM Co., Armonk, NY, USA).

A total of 111 patients were included (45 were on NOACs and 66 on warfarin). Diagnosis was confirmed by magnetic resonance imaging (MRI)/magnetic resonance venography (MRV) in 96, CT venogram in 10 and both MRV and CT venogram in five patients. Out of 45 patients on NOACs, 36 were given rivaroxaban and nine were given dabigatran. Warfarin was adjusted to achieve an international normalized ratio (INR) of 2 to 3 . The mean age of the participants was $39.3 \pm 14.9$ years and $57 \%$ were females and the two groups were comparable (Table 1). Hematological disorders followed by systemic infections were the most common etiological factors for CVT in both the NOAC and warfarin groups. Malignancy was a more common predisposing factor in the warfarin group, with six out of 66 patients suffering from the condition. No patient in the NOAC arm had malignancy.

Whereas the two groups did not differ significantly in terms of clinical features, patients in the NOAC group presented more frequently with headache ( $93 \%$ vs. $63 \%)$ and were significantly more likely than warfarin group to have intracerebral hemorrhage on initial imaging, reflecting the comfort of treating physician with NOACs. There were no other significant differences in the clinical and imaging features of the two groups. Patients in both groups were started on oral anticoagulation (warfarin with target INR 2 to 3 ; rivaroxaban 15 to $20 \mathrm{mg}$; dabigatrin 75 to $150 \mathrm{mg}$ twice a day) within median of one week of diagnosis. At discharge as well as at 6 months followup, the functional outcomes as measured by modified Rankin Scale, were not significantly different between the two groups. Four patients died during hospital stay, one in the NOAC arm and three in the warfarin arm. The hospital course was complicated by extracranial hemorrhage in two patients, both of whom were in the warfarin group. Two additional patients were dead at 6 months follow-up, one in each group.

Follow-up ranged from minimum 6 to 13 months (median, 8). All patients underwent neurological examination at followup. Follow-up evaluation included history of headaches, seizures, any signs of bleeding, side effects and compliance of medications, evaluation of modified Rankin Scale score and any imaging. At 6 months follow-up (95/107 discharged patients, 89\%), clinical neurological worsening was reported in one patient (warfarin group), and no patient required switching from one to the other group. In addition, no patient required discontinuation of therapy by the physician. Only 12 patients (five in NOAC group, seven in warfarin group) had follow-up MRI/MRV (one patient for neurologic worsening, mostly for persistent headache and non-improvement) during 6 months follow-up. Of these, four in the NOAC arm and four in the warfarin arm had complete and the rest had partial recanalization.

Six patients reported systemic bleeding at follow-up, two in the NOAC arm and four in the warfarin arm. As per International Society on Thrombosis and Haemostasis (ISTH) classification, one patient had major, one non-major clinically relevant, and four had minor hemorrhages. Only one patient in the NOAC arm required blood transfusion and a single patient in the warfarin group had intracranial hemorrhage that required intervention. No recurrent thrombotic events were seen in any of the patients.

This open label, comparative study reports on the largest co- 
hort of CVT patients treated with new oral anticoagulants and shows that the use of NOACs (mainly rivaroxaban and dabigatran) appear to be safe and may be as effective as warfarin in patients with CVT. Limitations of our study include non-randomization, open label, lack of adequate radiological data at follow-up to assess recanalization and non-blinded and nonadjudicated outcome assessments. Despite these, our study findings provide external validity for the RESPECT CVT results in a real-world setting and supports the growing trend of NOAC use for this potentially devastating condition.

\section{References}

1. Luo $Y$, Tian $X$, Wang $X$. Diagnosis and treatment of cerebral venous thrombosis: a review. Front Aging Neurosci 2018;10:2.

2. Einhäupl KM, Villringer $A$, Meister W, Mehraein S, Garner $C_{1}$ Pellkofer $M$, et al. Heparin treatment in sinus venous thrombosis. Lancet 1991;338:597-600.

3. Nagaraja D, Rao BSS, Taly AB, Subhash MN. Randomized controlled trial of heparin in puerperal cerebral venous/sinus thrombosis. NIMHANS J 1995;13:111-115.

4. de Bruijn SF, Stam J. Randomized, placebo-controlled trial of anticoagulant treatment with low-molecular-weight heparin for cerebral sinus thrombosis. Stroke 1999;30:484-488.

5. Linkins LA, Choi PT, Douketis JD. Clinical impact of bleeding in patients taking oral anticoagulant therapy for venous thromboembolism: a meta-analysis. Ann Intern Med 2003;139:893900.

6. Fang MC, Go AS, Chang Y, Hylek EM, Henault LE, Jensvold $N G$, et al. Death and disability from warfarin-associated intracranial and extracranial hemorrhages. Am J Med 2007; 120:700-705.
7. Chai-Adisaksopha $C_{1}$ Crowther M, Isayama T, Lim W. The impact of bleeding complications in patients receiving targetspecific oral anticoagulants: a systematic review and metaanalysis. Blood 2014;124:2450-2458.

8. Hon SF, Li HL, Cheng PW. Use of direct thrombin inhibitor for treatment of cerebral venous thrombosis. J Stroke Cerebrovasc Dis 2012;21:915.e11-915.e15.

9. Rao SK, Ibrahim M, Hanni CM, Suchdev K, Parker D, Raja-

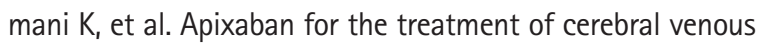
thrombosis: a case series. J Neurol Sci 2017;381:318-320.

10. Anticoli S, Pezzella FR, Scifoni G, Ferrari C, Pozzessere C. Treatment of cerebral venous thrombosis with rivaroxaban. $J$ Biomedical Sci 2016;5:3.

11. Mendonça MD, Barbosa R, Cruz-e-Silva V, Calado S, VianaBaptista M. Oral direct thrombin inhibitor as an alternative in the management of cerebral venous thrombosis: a series of 15 patients. Int J Stroke 2015;10:1115-1118.

12. Ferro JM, Dentali F, Coutinho JM, Kobayashi A, Caria J, Desch $M$, et al. Rationale, design, and protocol of a randomized controlled trial of the safety and efficacy of dabigatran etexilate versus dose-adjusted warfarin in patients with cerebral venous thrombosis. Int J Stroke 2018;13:766-770

Correspondence: Mohammad Wasay

Department of Neurology, Aga Khan University, Stadium Road, Karachi 74800, Pakistan

Tel: $+92-21-4930051$

Fax: +92-21-34934294

E-mail: mohammad.wasay@aku.edu

Received: January 15, 2019

Revised: February 21, 2019

Accepted: March 10, 2019

The authors have no financial conflicts of interest. 